\title{
The law of genetic inheritance
}

\author{
Zhe Yin ${ }^{1,2}$ \\ ${ }^{1}$ Mathematics Department, Yanbian University, Yanji, China \\ ${ }^{2}$ Department of Information Management, Peking University, Beijing, China \\ Email: yinzhe@ybu.edu.cn
}

Received 13 December 2011; revised 17 January 2012; accepted 6 February 2012

\begin{abstract}
In this paper, based on binomial formula, a law of genetic inheritance is proposed, and it would have certain reference value for future genetic inheritance research. Besides, a recursive algorithm for recessive inheritance is also proposed. Given any two of the male parent, female parent and filial generation, the genomic information of the third one can be calculated uniquely.
\end{abstract}

Keywords: Gene; Inheritance Law; Inheritability Ratio

\section{INTRODUCTION}

Human beings has experienced tens of thousands of generations since its born 5 million years ago. The role of genome has been acknowledged worldwide. This paper will present a complete set of genetic inheritance law, and a meaningful recursive algorithm in the delivery of genome information.

\section{BASIC ASSUMPTION}

Genetic Behavior is divided into dominant inheritance and recessive inheritance. Assume that $D$ represents for dominant inheritance factor in offspring, and $d$ represents for recessive inheritance factor in offspring. Two inheritance factors can be dependent or independent. Dependent means there is cross effect between $D$ and $d$, that is, $(D d)$ is also an inheritance result.

The possible result is $D$ or $d$ when the inheritance factors of male parent and female parent are independent, and the possible result can be $D, d,(D d)^{+},(D d)^{-}$, $D(D d)^{+}, d(D d)^{+}, D(D d)^{-}$, or $(D d)^{-} d$ when they are dependent.

Binomial formula: $(D+d)^{n}=\sum_{i=0}^{n} C_{n}^{i} D^{i} d^{n-i}$, where $C_{n}^{i}=\frac{n !}{i !(n-i) !}$

Coefficients of the odd and even items in the expansion are equal.
The expansion $(D+d)^{n}=\sum_{i=0}^{n} C_{n}^{i} D^{i} d^{n-i}$ is just all possible outcomes of the $(n-1)$-th generation of female and male parents hybrid.

\section{TWO LEMMAS}

Lemma 1: When the female parent and male parent are dominant inheritance, the inheritance factors will fit the following addition principle 1 :

$$
\begin{aligned}
& D \oplus D=D ; \\
& D \oplus d=D ; \\
& d \oplus D=D ; \\
& d \oplus d=d .
\end{aligned}
$$

Lemma 2: When the female parent and male parent are recessive inheritance, the inheritance factors will fit the following addition principle 2:

$$
\begin{aligned}
& D \oplus D=d ; \\
& D \oplus d=D ; \\
& d \oplus D=D ; \\
& d \oplus d=d .
\end{aligned}
$$

\section{GENETIC INHERTANCE LAW}

Genetic inheritance law:

1) All possible inheritance results of the $(n-1)$-th generation of female and male parents hybrid will fit the following expansion:

$$
\begin{aligned}
(D+d)^{n} & =\sum_{i=0}^{n} C_{n}^{i} D^{i} d^{n-i} \\
& =C_{n}^{0} D^{n}+C_{n}^{1} D^{n-1} d+C_{n}^{2} D^{n-2} d^{2}+\cdots+C_{n}^{n} d^{n},
\end{aligned}
$$

where $C_{n}^{i}=\frac{n !}{i !(n-i) !}$;

2) Inheritance factors meet distributive law (separate regularity);

3) Inheritance factors meet associative law (law of independent assortment); 
4) Dominant inheritance meets addition principle 1;

5) Recessive inheritance meets addition principle 2;

6) When $D$ and $d$ are independent, the possible result is $D$ or $d$;

7) When $D$ and $d$ are dependent, there will be cross effect between them, $(D d)$ will be regarded as inheritance factor which also have dominant inheritance $(D d)^{+}$and recessive inheritance $(D d)^{-}$(linkage inheritance rule), and possible result is: $D, d,(D d)^{+},(D d)^{-}, D(D d)^{+}$, $d(D d)^{+}, D(D d)^{-}$, or $(D d)^{-} d$.

Note: when $n=2$, this inheritance law is just Mendel's law [1].

\section{INFERENCES OF THE INHERITANCE LAW}

Inference 1: When meets dominant inheritance and $D$ and $d$ are independent, the 1st generation (1st) will be:

$$
(D+d)^{2}=D^{2}+2 D d+d^{2}=3 D+d
$$

the 2nd generation (2nd) will be:

$$
(D+d)^{3}=D^{3}+3 D^{2} d+3 D d^{2}+d^{3}=7 D+d
$$

the 3rd generation (3rd) will be:

$$
(D+d)^{4}=D^{4}+4 D^{3} d+6 D^{2} d^{2}+4 D^{1} d^{3}+d^{4}=15 D+d
$$

the $(n-1)$-th generation $((n-1)-$ th) will be:

$$
\begin{aligned}
(D+d)^{n} & =C_{n}^{0} D^{n}+C_{n}^{1} D^{n-1} d+C_{n}^{2} D^{n-2} d^{2}+\cdots+C_{n}^{n} d^{n} \\
& =\left(2^{n}-1\right) D+d(\text { see Table } 1) .
\end{aligned}
$$

Inference 2: When meets recessive inheritance and $D$ and $d$ are independent, the 1st generation (1st) will be:

$$
(D+d)^{2}=D^{2}+2 D d+d^{2}=2 D+2 d ;
$$

the 2nd generation (2nd) will be:

$$
(D+d)^{3}=D^{3}+3 D^{2} d+3 D d^{2}+d^{3}=4 D+4 d ;
$$

the 3rd generation (3rd) will be:

$$
(D+d)^{4}=D^{4}+4 D^{3} d+6 D^{2} d^{2}+4 D^{1} d^{3}+d^{4}=8 D+8 d
$$

the $(n-1)$-th generation $((n-1)-$ th) will be:

$$
\begin{aligned}
(D+d)^{n} & =C_{n}^{0} D^{n}+C_{n}^{1} D^{n-1} d+C_{n}^{2} D^{n-2} d^{2}+\cdots+C_{n}^{n} d^{n} \\
& =2^{n-1} D+2^{n-1} d \text { (see Table 2). }
\end{aligned}
$$

Inference 3: When meets dominant inheritance, $D$ and $d$ are dependent, and $(D d)^{+}$is dominant inheritance, the 1st generation (1st) will be:

$$
(D+d)^{2}=D^{2}+2(D d)^{+}+d^{2}=D+2(D d)^{+}+d
$$

the 2nd generation (2nd) will be:

$$
\begin{aligned}
(D+d)^{3} & =D^{3}+3 D^{2} d+3 D d^{2}+d^{3} \\
& =D+3 D(D d)^{+}+3(D d)^{+} d+d
\end{aligned}
$$

the 3rd generation (3rd) will be:

$$
\begin{aligned}
(D+d)^{4} & =D^{4}+4 D^{3} d+6 D^{2} d^{2}+4 D^{1} d^{3}+d^{4} \\
& =D+4 D(D d)^{+}+6(D d)^{+}+4(D d)^{+} d+d
\end{aligned}
$$

the 4th generation (4th) will be:

$$
\begin{aligned}
(D+d)^{5} & =D^{5}+5 D^{4} d+10 D^{3} d^{2}+10 D^{2} d^{3}+5 D d^{4}+d^{5} \\
& =D+15 D(D d)^{+}+15(D d)^{+} d+d \text { (see Table 3). }
\end{aligned}
$$

Inference 4: When meets dominant inheritance, $D$ and $d$ are dependent, and $(D d)^{+}$is recessive inheritance, the 1 st generation (1st) will be:

$$
(D+d)^{2}=D^{2}+2(D d)^{-}+d^{2}=D+2(D d)^{-}+d
$$

the 2nd generation (2nd) will be:

$$
\begin{aligned}
(D+d)^{3} & =D^{3}+3 D^{2} d+3 D d^{2}+d^{3} \\
& =D+3 D(D d)^{-}+3(D d)^{-} d+d
\end{aligned}
$$

the 3rd generation (3rd) will be:

$$
\begin{aligned}
(D+d)^{4} & =D^{4}+4 D^{3} d+6 D^{2} d^{2}+4 D^{1} d^{3}+d^{4} \\
& =D+4 D(D d)^{-}+6(D d)^{-}+4(D d)^{-} d+d
\end{aligned}
$$

the 4th generation (4th) will be:

$$
\begin{aligned}
(D+d)^{5} & =D^{5}+5 D^{4} d+10 D^{3} d^{2}+10 D^{2} d^{3}+5 D d^{4}+d^{5} \\
& =D+15 D(D d)^{-}+15(D d)^{-} d+d \text { (see Table 4). }
\end{aligned}
$$

Inference 5: When meets recessive inheritance, $D$ and $d$ are dependent, and $(D d)^{+}$is dominant inheritance, the 1st generation (1st) will be:

$$
(D+d)^{2}=D^{2}+2 D d+d^{2}=2(D d)^{+}+2 d ;
$$

the 2nd generation (2nd) will be:

$$
\begin{aligned}
(D+d)^{3} & =D^{3}+3 D^{2} d+3 D d^{2}+d^{3} \\
& =(D d)^{+}+3 D(D d)^{+}+3(D d)^{+} d+d ;
\end{aligned}
$$

the 3rd generation (3rd) will be:

$$
\begin{aligned}
(D+d)^{4} & =D^{4}+4 D^{3} d+6 D^{2} d^{2}+4 D^{1} d^{3}+d^{4} \\
& =7(D d)^{+}+8(D d)^{+} d+d
\end{aligned}
$$

the 4th generation (4th) will be:

$$
\begin{aligned}
& (D+d)^{5}=D^{5}+5 D^{4} d+10 D^{3} d^{2}+10 D^{2} d^{3}+5 D d^{4}+d^{5} \\
& =5(D d)^{+}+10 D(D d)^{+}+16(D d)^{+} d+d \text { (see Table 5). }
\end{aligned}
$$

Inference 6: When meets recessive inheritance, $D$ and $d$ are dependent, and $(D d)^{+}$is recessive inheritance, the 1st generation (1st) will be: 
Table 1. Inheritability ratio.

\begin{tabular}{|c|c|c|c|c|c|c|c|c|}
\hline Generation $\quad$ Inheritance factor & $D$ & $d$ & $(D d)^{+}$ & $(D d)^{-}$ & $D(D d)^{+}$ & $d(D d)^{+}$ & $D(D d)^{-}$ & $(D d)^{-} d$ \\
\hline 1st & $3 / 4$ & $1 / 4$ & & & & & & \\
\hline 2nd & $7 / 8$ & $1 / 8$ & & & & & & \\
\hline 3rd & $15 / 16$ & $1 / 16$ & & & & & & \\
\hline$(n-1)$-th & $\left(2^{n}-1\right) / 2^{n}$ & $1 / 2^{n}$ & & & & & & \\
\hline
\end{tabular}

Table 2. Inheritability ratio.

\begin{tabular}{|c|c|c|c|c|c|c|c|c|}
\hline Generation $\quad$ Inheritance factor & $D$ & $d$ & $(D d)^{+}$ & $(D d)^{-}$ & $D(D d)^{+}$ & $d(D d)^{+}$ & $D(D d)^{-}$ & $(D d)^{-} d$ \\
\hline 1st & $1 / 2$ & $1 / 2$ & & & & & & \\
\hline 2nd & $1 / 2$ & $1 / 2$ & & & & & & \\
\hline 3rd & $1 / 2$ & $1 / 2$ & & & & & & \\
\hline$(n-1)-t h$ & $1 / 2$ & $1 / 2$ & & & & & & \\
\hline
\end{tabular}

Table 3. Inheritability ratio.

\begin{tabular}{|c|c|c|c|c|c|c|c|c|}
\hline Generation $\quad$ Inheritance factor & $D$ & $d$ & $(D d)^{+}$ & $(D d)$ & $D(D d)^{+}$ & $d(D d)^{+}$ & $D(D d)^{-}$ & $(D d)^{-} d$ \\
\hline $1 \mathrm{st}$ & $1 / 4$ & $1 / 4$ & $1 / 2$ & & & & & \\
\hline 2nd & $1 / 2$ & $1 / 2$ & $3 / 4$ & & $\begin{array}{l}\text { 3/8 When } \\
\text { interactive }\end{array}$ & $\begin{array}{l}\text { 3/8 When } \\
\text { interactive }\end{array}$ & & \\
\hline 3rd & $5 / 16$ & $5 / 16$ & $3 / 8$ & & $\begin{array}{l}\text { 1/4 When } \\
\text { interactive }\end{array}$ & $\begin{array}{l}\text { 1/4 When } \\
\text { interactive }\end{array}$ & & \\
\hline 4th & $1 / 2$ & $1 / 2$ & $15 / 16$ & & $\begin{array}{l}\text { 15/32 When } \\
\text { interactive }\end{array}$ & $\begin{array}{l}\text { 15/32 When } \\
\text { interactive }\end{array}$ & & \\
\hline
\end{tabular}

Table 4. Inheritability ratio.

\begin{tabular}{|c|c|c|c|c|c|c|c|c|}
\hline Generation Inheritance factor & $D$ & $d$ & $(D d)^{+}$ & $(D d)^{-}$ & $D(D d)^{+}$ & $d(D d)^{+}$ & $D(D d)^{-}$ & $(D d)^{-} d$ \\
\hline $1 \mathrm{st}$ & $1 / 4$ & $1 / 4$ & & $1 / 2$ & & & & \\
\hline 2nd & $1 / 2$ & $1 / 2$ & & & & & $\begin{array}{l}\text { 3/8 When } \\
\text { interactive }\end{array}$ & $\begin{array}{l}\text { 3/8 When } \\
\text { interactive }\end{array}$ \\
\hline 3rd & $5 / 16$ & $5 / 16$ & & $3 / 8$ & & & $\begin{array}{l}\text { 1/4 When } \\
\text { interactive }\end{array}$ & $\begin{array}{l}1 / 4 \text { When } \\
\text { interactive }\end{array}$ \\
\hline 4th & $1 / 2$ & $1 / 2$ & & & & & $\begin{array}{l}\text { 15/32 When } \\
\text { interactive }\end{array}$ & $\begin{array}{l}15 / 32 \text { When } \\
\text { interactive }\end{array}$ \\
\hline
\end{tabular}

Table 5. Inheritability ratio.

\begin{tabular}{|c|c|c|c|c|c|c|c|c|c|}
\hline Generation & Inheritance factor & $D$ & $d$ & $(D d)^{+}$ & $(D d)^{-}$ & $D(D d)^{+}$ & $d(D d)^{+}$ & $D(D d)^{-}$ & $(D d)^{-} d$ \\
\hline & $1 \mathrm{st}$ & & $1 / 2$ & $1 / 2$ & & & & & \\
\hline & 2nd & $3 / 8$ & $1 / 2$ & $7 / 8$ & & $\begin{array}{l}\text { 3/8 When } \\
\text { interactive }\end{array}$ & $\begin{array}{l}\text { 3/8 When } \\
\text { interactive }\end{array}$ & & \\
\hline & 3rd & & $9 / 16$ & $15 / 16$ & & & $\begin{array}{l}1 / 2 \text { When } \\
\text { interactive }\end{array}$ & & \\
\hline & 4th & $5 / 16$ & $17 / 32$ & $31 / 32$ & & $\begin{array}{l}\text { 5/16 When } \\
\text { interactive }\end{array}$ & $\begin{array}{l}1 / 2 \text { When } \\
\text { interactive }\end{array}$ & & \\
\hline
\end{tabular}


Table 6. Inheritability ratio.

\begin{tabular}{|c|c|c|c|c|c|c|c|c|}
\hline Generation $\quad$ Inheritance factor & $D$ & $d$ & $(D d)^{+}$ & $(D d)^{-}$ & $D(D d)^{+}$ & $d(D d)^{+}$ & $D(D d)^{-}$ & $(D d)^{-} d$ \\
\hline $1 \mathrm{st}$ & & $1 / 2$ & & $1 / 2$ & & & & \\
\hline 2nd & $3 / 8$ & $1 / 2$ & & $7 / 8$ & & & $\begin{array}{l}\text { 3/8 When } \\
\text { interactive }\end{array}$ & $\begin{array}{l}\text { 3/8 When } \\
\text { interactive }\end{array}$ \\
\hline 3rd & & $9 / 16$ & & $15 / 16$ & & & & $\begin{array}{l}1 / 2 \text { When } \\
\text { interactive }\end{array}$ \\
\hline 4th & $5 / 16$ & $17 / 32$ & & $31 / 32$ & & & $\begin{array}{l}\text { 5/16 When } \\
\text { interactive }\end{array}$ & $\begin{array}{l}1 / 2 \text { When } \\
\text { interactive }\end{array}$ \\
\hline
\end{tabular}

$$
(D+d)^{2}=D^{2}+2 D d+d^{2}=2(D d)^{-}+2 d ;
$$

the 2nd generation (2nd) will be:

$$
\begin{aligned}
(D+d)^{3} & =D^{3}+3 D^{2} d+3 D d^{2}+d^{3} \\
& =(D d)^{-}+3 D(D d)^{-}+3(D d)^{-} d+d
\end{aligned}
$$

the 3rd generation (3rd) will be:

$$
\begin{aligned}
(D+d)^{4} & =D^{4}+4 D^{3} d+6 D^{2} d^{2}+4 D^{1} d^{3}+d^{4} \\
& =7(D d)^{-}+8(D d)^{-} d+d
\end{aligned}
$$

the 4th generation (4th) will be:

$$
\begin{aligned}
& (D+d)^{5}=D^{5}+5 D^{4} d+10 D^{3} d^{2}+10 D^{2} d^{3}+5 D d^{4}+d^{5} \\
& =5(D d)^{-}+10 D(D d)^{-}+16(D d)^{-} d+d .(\text { see Table 6). }
\end{aligned}
$$

\section{RECURSIVE NATURE OF GENETIC ALGORITHM FOR RECESSIVE INHERITANCE}

From lemma 2, we can know such addition principle:

$$
\begin{aligned}
& D \oplus D=d, \\
& D \oplus d=D, \\
& d \oplus D=D,
\end{aligned}
$$

and

$$
d \oplus d=d \text {. }
$$

These addition principles meet orthogonality, independence and uniqueness.
This principle gives a closure algorithm, that is, the genome information of filial generation won't change when compared with male parent and female parent; all genome information of filial generation will be influenced by the parents'; and, given any two of the male parent, female parent and filial generation, the genomic information of the third one can be calculated uniquely. Above is the recursive genetic algorithm for recessive inheritance.

\section{CONCLUSIONS}

We think that the genetic inheritance law proposed in this paper would have certain reference value for future genetic inheritance research. Using clinical inheritance statistic information can easily get similar conclusions. We have used clinical genetic disease statistics, such as schizophreniform psychosis, verified the rationality of the law. Besides, in mathematics, the binomial theorem has been proved to be unquestionable axiom. The inheritance rule of a variety of parents can be obtained easily by $(D+d)^{m}(Y+y)^{n}$.

Gene is under the influence of the living environment, not set in stone. How to quantify the specific changes and how to determine the timing of gene mutation need further study in future.

\section{REFERENCE}

[1] Plomin, R. (2008) Behavior genetics, East China Normal University Press, Shanghai. 